POS PROCEEDINGS

\title{
Study of persistent VHE gamma-ray emission detected with the MAGIC telescopes from PKS 1510-089, during low flux states detected by Fermi-LAT
}

\section{J. Becerra González}

Universidad de La Laguna, Dpto. Astrofísica, E-38206 La Laguna, and Inst. de Astrofísica de Canarias, E-38200 La Laguna, Tenerife, Spain

E-mail: jbecerragonzalez@gmail.com

J. Sitarek

University of Łódź, Department of Astrophysics, PL-90236 Łódź, Poland

C. Nigro

Deutsches Elektronen-Synchrotron (DESY), D-15738 Zeuthen, Germany

V. Fallah Ramazani

Finnish Centre of Astronomy with ESO (FINCA), University of Turku, FI-20014 Turku, Finland

E. Lindfors

Finnish Centre of Astronomy with ESO (FINCA), University of Turku, FI-20014 Turku, Finland

F. Tavecchio

Osservatorio di Brera, Milan and National Institute for Astrophysics (INAF), I-00136 Rome, Italy

for the MAGIC'and Fermi-LAT Collaborations

For collaboration list see PoS(ICRC2019)1177 
PKS 1510-089 is one of the few Flat Spectrum Radio Quasars (FSRQs) detected in the very-high-energy (VHE, E $>100 \mathrm{GeV}$ ) gamma-ray band. FSRQs can only be detected in the VHE band typically during flaring state. It is due to their low energy peaking spectral energy distributions (SEDs) w.r.t. BL Lac objects, and their steep gamma-ray spectra (which could even include some intrinsic absorption). During flaring states, typically not only the fluxes are enhanced but also their SED peaks are shifted towards higher energies, facilitating the detection in the VHE band with Cherenkov telescopes. The FSRQ VHE family is only limited to seven sources (TeVCat [1]), and their discovery in the VHE band challenges the theoretical emission scenarios. Up to now, such studies were biased towards flaring states. In this work, we present the first study of a FSRQ showing persistent emission outside of the flaring states.

The MAGIC telescopes have monitored this source regularly since 2012. In order to select the periods when the source was not highly active, the Fermi-LAT gamma-ray survey data above $1 \mathrm{GeV}$ have been used. A flux threshold of $3 \times 10^{-8} \mathrm{ph} \mathrm{cm}^{-2} \mathrm{~s}^{-1}$ at energies above $1 \mathrm{GeV}$ was set as definition of the low gamma-ray activity state. Based on such selection criteria, $75 \mathrm{~h}$ of VHE data from the MAGIC telescopes was selected for this analysis taken from 2012 to 2017. The VHE low-state data sample yields a significant detection at the level of $9.5 \sigma$. The measured flux is smaller by a factor of 80 times w.r.t. the highest flux observed from this object, $(4.3 \pm 0.6) \times 10^{-12} \mathrm{phcm}^{-2} \mathrm{~s}^{-1}$ above $150 \mathrm{GeV}$. The spectral shape however, even during low state, is consistent with the one observed from PKS 1510-089 during flaring states.

The MWL SED during the low gamma-ray state is built from radio to the VHE band and modeled within the External Compton framework. No absorption features are detected in the GeV-VHE gamma-ray regime, constraining the location of the emitting region beyond the broad line region (BLR) to allow the VHE photons to escape unattenuated. Therefore, the target photons for the inverse Compton scattering correspond to the IR photons from the torus. This is the first time that constraints on the location of the emitting region within the relativistic jets of FSRQs during no flaring states can be derived. Such constraint is compatible with the previous findings from the VHE FSRQ family detected during high states. All the details on the analysis and results can be found in MAGIC Collaboration et al. 2018 [2].

36th International Cosmic Ray Conference -ICRC2019-

July 24th - August 1st, 2019

Madison, WI, U.S.A.

\footnotetext{
${ }^{*}$ Speaker.

†https: / /magic.mpp.mpg.de/
} 


\section{Acknowledgements}

We would like to thank the Instituto de Astrofísica de Canarias for the excellent working conditions at the Observatorio del Roque de los Muchachos in La Palma. The financial support of the German BMBF and MPG, the Italian INFN and INAF, the Swiss National Fund SNF, the ERDF under the Spanish MINECO (FPA2015-69818-P, FPA2012-36668, FPA2015-68378-P, FPA2015-69210-C6-2-R, FPA2015-69210-C6-4-R, FPA2015-69210-C6-6-R, AYA2015-71042-P, AYA2016-76012-C3-1-P, ESP2015-71662-C2-2-P, FPA2017?90566?REDC), the Indian Department of Atomic Energy, the Japanese JSPS and MEXT, the Bulgarian Ministry of Education and Science, National RI Roadmap Project DO1-153/28.08.2018 and the Academy of Finland grant nr. 320045 is gratefully acknowledged. This work was also supported by the Spanish Centro de Excelencia "Severo Ochoa" SEV-2016-0588 and SEV-2015-0548, and Unidad de Excelencia "María de Maeztu" MDM-2014-0369, by the Croatian Science Foundation (HrZZ) Project IP2016-06-9782 and the University of Rijeka Project 13.12.1.3.02, by the DFG Collaborative Research Centers SFB823/C4 and SFB876/C3, the Polish National Research Centre grant UMO2016/22/M/ST9/00382 and by the Brazilian MCTIC, CNPq and FAPERJ.

The Fermi LAT Collaboration acknowledges generous ongoing support from a number of agencies and institutes that have supported both the development and the operation of the LAT as well as scientific data analysis. These include the National Aeronautics and Space Administration and the Department of Energy in the United States, the Commissariat à l'Energie Atomique and the Centre National de la Recherche Scientifique / Institut National de Physique Nucléaire et de Physique des Particules in France, the Agenzia Spaziale Italiana and the Istituto Nazionale di Fisica Nucleare in Italy, the Ministry of Education, Culture, Sports, Science and Technology (MEXT), High Energy Accelerator Research Organization (KEK) and Japan Aerospace Exploration Agency (JAXA) in Japan, and the K. A. Wallenberg Foundation, the Swedish Research Council and the Swedish National Space Board in Sweden.

Additional support for science analysis during the operations phase is gratefully acknowledged from the Istituto Nazionale di Astrofisica in Italy and the Centre National d'Études Spatiales in France. This work performed in part under DOE Contract DE-AC02-76SF00515.

J. Becerra González acknowledges the support of the Viera y Clavijo program funded by ACIISI and ULL.

\section{References}

[1] http://tevcat.uchicago.edu

[2] MAGIC Collaboration et al. 2018, A\&A, 619, A159 